\title{
A Retrospective Analysis of Patients with Leiomyoma
}

\author{
Renuka J. ${ }^{1}$, Harrini K. ${ }^{2}$, Mehrunnisa Abdul Huq ${ }^{3}$, Sajitha C. K. ${ }^{4}$, Jamila Hameed ${ }^{5}$ \\ ${ }^{1}$ Department of Obstetrics and Gynaecology, Karuna Medial College, Palakkad, Kerala, India. \\ ${ }^{2}$ Department of Obstetrics and Gynaecology, Karuna Medial College, Palakkad, Kerala, India. \\ ${ }^{3}$ Department of Obstetrics and Gynaecology, Karuna Medial College, Palakkad, Kerala, India. \\ ${ }^{4}$ Department of Obstetrics and Gynaecology, Karuna Medial College, Palakkad, Kerala, India. \\ ${ }^{5}$ Department of Obstetrics and Gynaecology, Karuna Medial College, Palakkad, Kerala, India.
}

\section{ABSTRACT}

\section{BACKGROUND}

A study of 200 women with fibroids was conducted in southern most part of India, Kerala, in order to analyse the prevalence, clinical features and impact on life style.

\section{METHODS}

A group of 200 women in Karuna Medical College and Hospital, Kerala, were studied from the case sheet records during the period April 2019 - Feb 2020. Women included in the study were in the age group of 20 - 60 yrs. Different aspects like age, diet, BMI, family history, socio economic status, comorbidities, lifestyle, smoking history, surgical history, obstetric and menstrual history, pain and intra operative and post-operative findings were analysed.

\section{RESULTS}

Prevalence of uterine fibroids in the age group of $41-50$ yrs. is $52.5 \%$. Women with fibroids reported mostly with profuse menstrual bleeding like menorrhagia (44.5\%) and metrorrhagia (9\%). Pain was noted in $12.5 \%$ of women. Dysmenorrhoea was noted in $9 \%$ of women. Some women $(10 \%)$ had no impact on the lifestyle; they were not aware of the presence fibroids. Some $9 \%$ of women had dyspareunia, and infertility was noted in $15 \%$ of women. In some patients, even large fibroids (as big as 24 weeks size) produced no complaints.

\section{CONCLUSIONS}

Uterine fibroids sometimes even if they are humongous caused no pressure symptoms and pain. Site of the fibroid causes problems and it is not the size of fibroid. Sometimes even huge fibroids can be asymptomatic as seen in few cases.

\section{KEY WORDS}

Leiomyoma, Clinical Features, Prevalence, Impact on Lifestyle
Corresponding Author: Jamila Hameed, D66 Doctors Quarters, Karuna Medical College and Hospital, Palakkad, Kerala, India.

E-mail: jamilahameed@gmail.com

DOI: $10.14260 / j e m d s / 2020 / 407$

Financial or Other Competing Interests: None.

How to Cite This Article:

Renuka J, Harrini K, Huq MA, et al. A retrospective analysis of patients with leiomyoma. J. Evolution Med. Dent. Sci. 2020;9(25):1869-1873, DOI:
Submission 27-03-2020,

Peer Review 28-04-2020,

Acceptance 30-04-2020,

Published 22-06-2020. 


\section{BACKGROUND}

Leiomyomas are benign smooth muscle tumours arising from myometrium. Growth of the tumours is directly proportional to the oestrogen as it regresses in the menopause. About $20 \%$ to $80 \%$ of women develop fibroid by the age of 50 . Fibroids are more common in obese women.

Fibroids are round, well circumscribed and have a pseudo capsule. Size varies from seedlings, even microscopic to huge ones. Cut section is whorl shape. There is a very rare syndrome called Pseudo Meigs's Syndrome when there is presence of fibroid with ascites and right sided hydrothorax. Reed Syndrome is a hereditary leiomyomatosis with renal cell carcinoma. Rarely we come across fibroids in the round ligament, uterosacral ligament and broad ligament. Sometimes it is noted rarely invading the vascular structures like intravenous leiomyomatosis, presence of fibroids in different parts of the body- benign metastasizing fibroids in lymph node, lung.

Fibroid may coexist with adenomyosis, endometriosis and salpingo-oophoritis. Anaemia may result because of the heavy menstrual bleeding in the presence of the fibroid. Hydroureter, hydronephrosis are the results of the pressure effect of the fibroids. This depends on the site of the fibroid mostly by the presence of fibroid in the lower uterine segment and cervical fibroid.

There are certain changes which the fibroid undergoes, namely atrophy, degeneration, torsion, intra capsular haemorrhage, infection, necrosis and malignancy. Chance of malignancy is very rare. It is only $0.1 \%$ incidence. Suspicion of malignancy is raised when the fibroid increases in size suddenly, pseudo capsule lost; there are plenty of necrotic and haemorrhagic tissues, especially in a post-menopausal woman, there is a sudden onset of pain and bleeding.

Degeneration of the fibroid comes in different flavours. Hyaline degeneration is common $60 \%$. Cystic degeneration, fatty degeneration, calcification is noted in post-menopausal women, appearance of womb-stone is noted.

Red degeneration of the fibroid occurs in second half of pregnancy wherein the pregnant lady comes with sudden pain, fever and tender abdomen. It is mistaken for acute abdomen, premature labour or accidental haemorrhage. Blood reports may reveal leucocytosis and raised ESR. This only needs a conservative management with symptomatic management, no surgery is needed. Pathology is attributed to the thrombosis of the vessels supplying the fibroid which is followed by the escape of "haem" pigment of the blood into the fibroid giving a red colour, hence the name. It is fishy in odour and noted as salmon pink in colour.

Incidence of fibroid is related to the family history, early menarche, nulliparity and obesity. ${ }^{1}$

Huge fibroids are asymptomatic and remain undiagnosed in our rural women. ${ }^{2}$ commonest menstrual symptoms associated with fibroids are menometrorrhagia. Menorrhagia, heavy menstrual flow is common association with intramural and submucous fibroid. Cervical fibroid, broad ligament fibroid and subserous fibroids are not associated with menstrual symptoms. Dysmenorrhea and pain may be noted in the submucous fibroid, cervical fibroids, and intramural fibroids. Pedunculated fibroids may undergo torsion, fibroid polyp cause pain.
Metrorrhagia is seen in cervical fibroid, fibroid polyp and submucous fibroid when there is super added infection. In other cases, the usual symptoms are menstrual heavy flow. ${ }^{3}$ torsion occurs in fibroid wherein it is subserous with a pedicle. Sometimes it can get detached from the uterus the mother from its origin and get blood supply from the neighbouring structures, becoming a parasitic fibroid. ${ }^{4}$

Submucous fibroids when attached to the fundus of the uterus may drag on the uterine fundus and cause chronic inversion of uterus which may look like a mass protruding through the vagina mimic prolapse. ${ }^{5}$

When cervical fibroids are present it cause infertility, dyspareunia, pressure symptoms on bladder and rectum. Patients come with retention of urine.

Central cervical fibroid when enlarged it sits on the uterus giving the appearance of the Dome of "St. Pauls" cathedral. Surgery of the cervical fibroid is a herculean task as it alters the anatomy of the course of the ureter, hence IVP and ureteric stenting is needed prior to surgery. ${ }^{6}$

Dyspareunia is noted in some women. Back pain is due to pressure on the nerves. Especially huge fibroids may cause difficulty in movement. ${ }^{7}$

Polycythaemia is a rare finding associated with broad ligament fibroid. True broad ligament fibroid arising from the broad ligament and growing towards the uterus push the ureter medially, while the pseudo broad ligament fibroids originating from the uterus push the ureter laterally when extending into the broad ligament. ${ }^{8}$ So, it is essential to know the anatomy of the ureter course while doing the surgery of the broad ligament. Urologist opinion, IVP, and ureteric stenting may be required prior to surgery.

There are plenty of options as for as the treatment is concerned. There are medical, surgical, minimal invasive and non-invasive methods. Treatment of fibroid is asymptomatic in $50 \%$ of the women. Medical treatment is suitable for younger patients when it is not very big. Size less than $5 \mathrm{~cm}$. Classical treatment for fibroid is the usage of OCP, progesterone, LNG, intra uterine device, GnRH, selective progesterone receptor modulators, ulipristal acetate and NSAIDs, raloxifene, Tamoxifen (SERM). In case of patients in the AUB and pelvic pain treatment of choice is Aromatase inhibitors and mifepristone, RU 486. ${ }^{9}$ Expectant management is the best choice for women approaching menopause. In such patients, anyway the risk of leiomyosarcoma is rare. This can be ruled out by MRI and estimation of lactate dehydrogenase in the follow up. ${ }^{10}$

\section{METHODS}

Details of a total of 200 women were collected from the case sheets. Hence the sample size is 200 . Retrospectively analysed from April 2019 to February 2020. Duration of study 11 months. Data collection was done from the case sheets from the medical report department. Ethical committee consent was taken and informed consent for investigations ordered were already obtained during consultation. Study design is a retrospective descriptive study with an aim to study the prevalence, associated risk factors, clinical features, age, BMI and ultrasound findings regarding the types of fibroids. Inclusion criteria were- women in the age group of 20 to 60 . For all women in this age group, case sheets were collected 
from medical records department. A manual collection of the data was done from tabulating each and every case sheet. A tedious attempt was made to study from these data collected to study the prevalence and establish information. It was found that in $10 \%$ of women huge fibroids came with no symptoms. Variables include age, socio economic status, family history, BMI, blood group, obstetrics and menstrual history, co morbidities, clinical findings. All investigations required for the patients, were completely studied and tabulated for any associated comorbidities. Exclusion criteria - the patients who had AUB, postmenopausal bleeding, endometrial carcinoma, leiomyosarcoma, other cervical pathology like ca cervix, CIN, and women above 60 years.

\section{Statistical Analysis}

Data was entered into Microsoft Excel and analysed using Epi Info software for statistical analysis.

\section{RESULTS}

A total of 200 women as per the inclusion criteria were enrolled in the study. Collection of the data for the study from case sheets was performed from the period April 2019 to February 2020. All the variables were standardised. Influence of high-risk factors namely age, BMI, family history, co morbidities, clinical symptoms and the impact on lifestyles were noted.

\section{Age}

Youngest patient in our group is 20 years whereas the oldest patient 60 years of age.

\section{BMI}

As far as the BMI is concerned $30 \%$ of patients are within normal weight $54 \%$ of patients are overweight $16 \%$ of patients are obese.

\section{Blood Group}

$54 \%$ patients are $0^{+v e}, 2.5 \%$ are $0^{-v e}, 19 \%$ are $A^{+v e}$, nil in $A^{-v e,}$ $16 \%$ are $\mathrm{B}^{+\mathrm{ve}}, 1.5 \%$ are $\mathrm{B}^{-\mathrm{ve}}, 7 \%$ are $\mathrm{AB}^{+\mathrm{ve}}$, nil in $\mathrm{AB}^{-\mathrm{ve}}$ blood group.

- Total number of women with symptoms of heavy menstrual bleeding is $44.5 \%$

- Total number of women with metrorrhagia is $9 \%$

Pain was noted and diagnosed as pressure symptoms on the bladder. About $9 \%$ women complain of dyspareunia. 15\% women complained infertility. $12.5 \%$ women complained of pain. Fibroids are asymptomatic in $10 \%$ of patients in our study.

Time old medical management is oral contraceptives. Sometimes pain killers and Ulipristal Acetate are used. Mirena was not used. Myomectomy was done in 5\%. Patients who have undergone myomectomy later underwent hysterectomy. Majority of the patients underwent surgical management.

\begin{tabular}{|ccc|}
\hline Age & Number of Patients & Percentage (\%) \\
$20-30$ & 25 & 12.5 \\
$31-40$ & 38 & 19 \\
$41-50$ & 105 & 52.5 \\
$51-60$ & 32 & 16 \\
Parity & Number of patients & Percentage (\%) \\
$1-3$ & 185 & 92.5 \\
$4-6$ & 15 & 7.5 \\
Comorbidities & Number of patients & Percentage \\
DM & 45 & 22.5 \\
HTN & 38 & 19 \\
Dyslipidemia & 16 & 8 \\
Bronchial asthma & 2 & 1 \\
Thyroid & 20 & 10 \\
Ca Breast & 6 & 3 \\
Family History & Number of patients & Percentage \\
Present & 142 & 71 \\
Not present & 58 & 29 \\
Total & $\mathbf{2 0 0}$ & \\
Table 1. Distribution of Patients of Leiomyoma According to Age, \\
Parity, Comorbidities, and Family History
\end{tabular}

\begin{tabular}{|ccc|}
\hline Number of Myomas & Number of Patients & Percentage (\%) \\
Solitary & 58 & 29 \\
$2-5$ & 108 & 54 \\
$6-10$ & 34 & 17 \\
Types of Myomas & Number of Patients & \\
Intramural & 168 & \\
Subserous & 60 & \\
Submucous & 55 & \\
Pedunculated & 15 & \\
Location of Myomas & Number of Patients & \\
Fundus & 78 & \\
Body & 136 & \\
Cervix & 9 & 12.5 \\
Broad Ligament & 8 & 27.5 \\
Fibroid polyp & 2 & 45 \\
Uterine Size & Number of Patients & \\
Uterus not palpable & 25 & \\
12 - 18 wk. size & 55 & \\
19-22 wk. size & 90 & \\
23 - 26 wk. size & 30 & \\
Table 2. Number, Type, Location and & \\
Uterine Size with Regard to Leiomyoma & \\
\hline
\end{tabular}

\begin{tabular}{|ccc|}
\hline Complications & No. of Patients & Percentage \\
Adhesion During Surgery & 21 & 10.5 \\
Bladder Injury & 1 & 0.5 \\
Bowel Injury & Nil & \\
No Adhesion & 178 & 89 \\
Total & 200 & \\
Treatment & No. of Patients & Percentage \\
Myomectomy & 10 & 5 \\
Total abdominal hysterectomy & 27 & 13.5 \\
Total abdominal hysterectomy with bilateral & 113 & 56.5 \\
$\begin{array}{c}\text { salpingo-oophorectomy } \\
\text { Total abdominal hysterectomy with Unilateral } \\
\text { salpingo-oophorectomy }\end{array}$ & 50 & 25 \\
\hline Table 3. Complications during Surgery and the Treatment \\
Given to the Patients with Leiomyoma \\
\hline
\end{tabular}

\begin{tabular}{|ccc|}
\hline Estimated Blood Loss & Number of Patients & Percentage \\
$<200 \mathrm{ml}$ & 163 & 81.5 \\
$201-500 \mathrm{ml}$ & 35 & 17.5 \\
$>500 \mathrm{ml}$ & 2 & 1 \\
Blood Transfusion & Number of Patients & Percentage (\%) \\
Preoperative & 66 & 33 \\
Intraoperative & 34 & 17 \\
Postoperative & 15 & 7.5 \\
No transfusion & 85 & 42.5 \\
Total & $\mathbf{2 0 0}$ \\
\hline \multicolumn{2}{|c|}{ Table 4. Pre-Operative and Post-Operative } \\
Transfusion Details \\
\hline
\end{tabular}

\begin{tabular}{|cc|}
\hline Post-Operative & \\
Superficial wound gap & 18 \\
Fever & 23 \\
No complication & 152 \\
Hospital Stay & Number of Patients \\
$3-5$ days & 160 \\
$6-8$ days & 24 \\
$>8$ days & 16 \\
Follow Up & No of Patients \\
Vault Prolapse & Nil \\
Pelvic abscess & 4 \\
Discharge & 16 \\
Bleeding & 8 \\
Dribbling of Urine & Nil \\
No complaint & 172 \\
HPE - Leiomyoma without any atypia & 200 \\
\hline Table 5. Post-Operative Complications and Hospital Stay of the \\
\end{tabular}


Total Hysterectomy was the treatment of choice in majority of the patients. patients who underwent hysterectomy- $95 \%$ in the age group of 40 to 60 Yrs. patients who had submucous fibroid $27.5 \%$, subserous fibroid $30 \%$, intramural fibroid $84 \%$. Only $10 \%$ of fibroid had no impact and $90 \%$ of fibroid had impact on the lifestyle.

\section{DISCUSSION}

Prevalence of Leiomyoma here is comparable to others and is increased by age and reaches maximum by 40 yrs. Similar to other studies, ${ }^{11}$ the mean age was 47 yrs. Menarche at an early age plays a risk role in the formation of fibroids, similar to oestrogenic nature of the breast and endometrial cancer.12 This is also shown in our study. Patients who were obese and over-weight had fibroids. Non-vegetarian diet seems to be having a risk in developing fibroids. Heavy menstrual bleeding and urinary ${ }^{13}$ symptoms which were noted among our patients were also reported in other studies. ${ }^{14}$ There are plenty of causes leading to menorrhagia in fibroids such as increase in bleeding surface, deficiency in contractility of uterus to control bleeding, the venous congestion, mechanical compression by venous stasis and due to endometrial hyperplasia which is associated with fibroid. Pain is a common symptom noted due to traction and increased intra-abdominal pressure. Diagnosis of the fibroid can be easily made by a bimanual vaginal examination. When the size is bigger than 16 weeks it is palpable per abdomen.

Signs on examination are that it is firm, movable from side to side, the lower border is not felt as it arises from the pelvis. Lateral borders are made out unless it is too huge it is not movable from side to side. When a bimanual examination is planned it is advisable to do a speculum examination, first a swab for culture and sensitivity and also a pap smear is taken.

Preformed bimanual palpation to explore the fornix to feel the fallopian tubes and ovaries. This may rule out the ovarian mass separate from the uterine mass wherein the groove is noted only if ovarian tumour is present. Hingorani sign is noted when done in Trendelenburg's position. Moving cervix, fibroid also moves but ovarian mass will not move. Position of the mass is also noteworthy. Fibroid usually causes a hypogastric swelling while the ovarian cyst occupies the iliac fossa. Fibroid is firm in consistency, will change when it has undergone cystic degeneration. Fluctuation and mobility is more in case of ovarian cyst.

\section{Limitations}

This study was done from the case records obtained from the Medical Record Department. Therefore, the study population can differ in comparison to the general women population. From the examination of the medical records the data, the clinical features, investigations and the diagnosis of the different types of fibroid, the operation notes, the intraoperative findings, post-operative complications and the histopathological findings are studied, and the results are well validated. Risk factors and prevalence were studied. Results of the study show that the reproductive age is under risk for developing fibroids. This is similar to the previous studies done. 15
Parity is of great importance for occurring fibroids. Nulliparity is a risk factor. It is noted in other papers. ${ }^{16}$ results showed that $5 \%$ of women had medical treatment, oral contraceptives and pain killers. These are useful as symptomatic drugs. In our patients few of them tried medical treatment. So, most of the patients opted for hysterectomy. So Total Hysterectomy was the main stay in the treatment of our patients.

\section{CONCLUSIONS}

Our study data on uterine fibroids is of great clinical importance as far as the women of this globe are concerned. Usually in this hurried, stressful mechanical life the women folks especially from the lower socio economical condition hardly find time and opportunity to take care of themselves in spite of all these short comings and there is negative impact on life. This is to be noted. A regular self-examination, free camps, master health check-ups including blood examination, Pap smear and ultrasound are mandatory for general screening of the population.

\section{ACKNOWLEDGEMENT}

We like to thank and show our gratitude to the following people for sharing their pearls of wisdom and moral support and tremendous help in the preparation of our manuscript.

1. Manager : Mr. Rahim

2. Principal : Dr. Vasanthamalai

3. Vice Principal : Dr. Seema Devi Patil

4. Ethical committee Karuna Medical College and staff of department of OBG.

\section{REFERENCES}

[1] Sparic R, Mirkovic L, Malvasi A, et al. Epidemiology of uterine myomas: a review. Int $\mathrm{J}$ Fertil Steril 2016;9(4):424-35.

[2] Divakar H. Asymptomatic uterine fibroids. Best Pract Res Clin Obstet Gynaecol 2008;22(4):643-54.

[3] Wegienka G, Baird DD, Hertz-Picciotto I, et al. Selfreported heavy bleeding associated with uterine leiomyomata. Obstet Gynecol 2003;101(3):431-7.

[4] Khan A, Shawl A, Leung PS. Parasitic leiomyoma of the greater omentum presenting as a small bowel obstruction. J Surg Case Rep 2018;2018(7):rjy164.

[5] Straub HL, Chohan L, Kilpatrick CC. Cervical and prolapsed submucosal leiomyomas complicating pregnancy. Obstet Gynecol Surv 2010;65(9):583-90.

[6] Patel P, Banker M, Munshi S, et al. Handling cervical myomas. J Gynecol Endose Surg 2011;2(1):30-2.

[7] Lippman SA, Warner M, Samuels S, et al. Uterine fibroids and gynecologic pain symptoms in a population-based study. Fertil Steril 2003;80(6):1488-94. 
[8] EI-Agwany AS. Huge broad ligament fibroid with paracervical extension: a safe approach by same setting. J Med Ultrasound 2018;26(1):45-7.

[9] Moroni RM, Vieira CS, Ferriani RA, et al. Pharmacological treatment of uterine fibroids. Ann Med Health Sci Res 2014;4(Suppl 3):S185-92.

[10] Moro E, Esposti ED, Borghese G, et al. The impact of hormonal replacement treatment in postmenopausal women with uterine fibroids: a state -of-the-art review of the literature. Medicina (Kaunas) 2019;55(9):549.

[11] Borgfeldt C, Andolf E. Transvaginal ultrasonographic findings in the uterus and the endometrium: low prevalence of leiomyoma in a random sample of women age 25-40 years. Acta Obstet Gynecol Scand 2000;79(3):202-7.
[12] Kim JJ, Sefton EC. Role of progesterone signaling in the pathogenesis of uterine leiomyoma. Mol Cell Endocrinol 2012;358(2):223-31.

[13] Gupta S, Jose J, Manyonda I. Clinical presentation of fibroids. Best Pract Res Clin Obstet Gynaecol 2008;22(4):615-26.

[14] Spies JB, Coyne K, Guaou NG, et al. The UFS-QOL, a new disease-specific symptom and health-related quality of life questionnaire for leiomyomata. Obstet Gynecol 2002;99(2):290-300.

[15] Parazzini F, Negri E, La Vecchia C, et al. Reproductive factors and risk of uterine fibroids. Epidemiology 1996;7(4):440-2.

[16] Parker WH. Etiology, symptomatology, and diagnosis of uterine myomas. Fertil Steril 2007;87(4):725-36. 